\title{
Versión española del cuestionario de Yesavage abreviado (GDS) para el despistaje de depresión en mayores de 65 años: adaptación y validación
}

\author{
J. Martínez de la Iglesia, Mạ C. Onís Vilches, R. Dueñas Herrero*, \\ C. Albert Colomer**, C. Aguado Taberné, R. Luque Luque** \\ M édico de Familia. U nidad Clínica de Occidente. Distrito Córdoba-Centro. Tutor U.D. \\ Medicina Familiar y Comunitaria de Córdoba. * Médico de Familia. Residente de \\ Psiquiatría. **Ár ea de Salud M ental. Hospital Universitario Reina Sofía. Córdoba
}

\section{RESUMEN}

Fundamento: existen escasos cuestionarios de cribado de depresíón adecuadamente validados al castellano.

Objetivo: adaptar y validar al castellano la ver sión abreviada de la Geriatric Depression Scale de Yesavage (GDS) para su uso en el cribado de de presiones en mayores de 65 años.

Diseño, material y método: el trabajo se ha realizado en consultas de un centro de salud de Aten ción Primaria mediante un estudio de validación de cuestionarios. Se ha realizado en tres etapas: en la primera se ha traducido y adaptado el cuestio nario al castellano (GDS-VE). En una segunda fase el médicolenfermera de cada sujeto aplicó el cuestionario de forma individual. Por último, otro investigador, de forma ciega, realizó al sujeto una entrevista semiestructurada para determinar sínto mas de depresión según los criterios establecidos en la CIE-10. Igualmente se realizó una evaluación del estado cognitivo y se cumplimentaron los cues tionarios de Montgomery-Asberg, Pfeiffer-VE, $M E C, I Q C O D E-V E$ y la parte de valoración de ac tividades de la vida diaria del OARS-MFAQ. El re sultado final de esta exploración fue considerado como el patrón oro de diagnóstico de depresión, siendo la referencia para el cálculo de la sensibili dad, especificidad y razones de máxima verosimili tud para un resultado positivo y negativo del test.

Resultados: se ha realizado la adaptación del $G D S$ obteniendo la versión a validar (GDS-VE). Finalizaron el estudio 249 personas mayores de 65 años, con media de edad de 74,3 años, el 67,5\%
The Spanish version of the Yesavage abbreviated questionnaire (GDS) to screen depressive dysfunc tions in patients older than 65 years

\section{ABSTRACT}

Background: there are few questionnaires of de pression screening appropriately validated in Spanish language.

Objective: to adapt and to validate to the Spa nish language the abbreviated version of the Ge riatric Depression Scale of Yessavage (GDS) for their use in the one sieved of depressive dysfunc tions in patients older than 65 years.

Design, material and method: the study was ca rried out in the consultations of a health center, in three stages: in the first one, the questionnaire was translated and adapted to the Spanish language (GDL-VE). In a second phase, the doctor or the nurse applied the questionnaire to every patient in dividually. Finally, another investigator carried out each a semiestructurated interview, in a blind way, to determine the depression symptoms according to the approaches settled down in the ICE-10. Cogni tive satate was also assesed, and the questionnaires of Montgomery-Asberg, Pfeiffer-VE, MEC, $I Q C O D E-E V$ were passed, as the part of the ques tionnaire OARS-MFAQ corresponding to the eva luation of the daily activities. The final result of this exploration was considered the gold standard for the diagnosis of depression, being the reference for the calculation of the sensibility, specificity and reasons of maximum verisimilitude for a positive and negative results of the test.

Results: an adaptation from the GDS to the ver sion GDS-GO was performed. Two hundred and forty-nine patients older than 65 years completed the study, with age of 74.3 on average, $67.5 \%$ of them 
mujeres y un $65 \%$ analfabetos o sin estudios. La fiabilidad intraobservador ha sido del 0,95 y la in terobservador del 0,65, con una consistencia inter na de 0,99. Se ha obtenido un área bajo la curva de 0,835. La sensibilidad alcanzada ha sido del $81,1 \%$ y la especificidad del $76,7 \%$ para un punto de corte de 5 o más puntos.

Conclusiones: los parámetros de fiabilidad y validez para la versión adaptada han resultado aceptables y similares a los del cuestionario origi nal. Se recomienda utilizar un punto de corte de 5 o más para considerar la posible existencia de depresión.

Palabras clave: Anciano. Depresión. Cribado. Validación. GDS. Cuestionario. were women and 65 were analphabet or with no stu dies. The intraobserver reliability was 0.95 and the interobserver was 0.65 , with an internal consistency of the 0.99. An under curve area of 0.385 was obtai ned. The sensibility reached $81.1 \%$ and the speci ficity $76.7 \%$, for a cut point of 5 or more.

Conclusions: the parameters of reliability and validity for the adapted version of the questionnaire have been acceptable and similar to those of the original questionnaire. It is recommended to use a cut point of 5 or above to consider the exis tence of a depression.

Key words: Aged people. Depression. Scree ning. Validation. GDS. Questionnaire.

\section{INTRODUCCIÓN}

La depresión constituye uno de los síndromes más frecuentes e incapacitantes de la población anciana, siendo su frecuencia variable según el contexto, si bien en todos ellos constituye un importante problema de salud ${ }^{1-4}$. Se ha establecido que los trastornos depresivos afectan a alrededor del $10 \%$ de los ancianos que viven en la comunidad, y entre el 15 y el $35 \%$ de los que viven en residencias $^{1}$.

La depresión en los mayores constituye un cuadro heterogéneo que presenta ciertas características diferenciales. Los factores psicosociales (dificultades económicas, aislamiento social, pérdida de seres queridos,...) juegan un papel más importante en su etiopatogenia que en edades más jóvenes. Además, los cambios biológicos propios del envejecimiento, la presencia de déficits cognitivos, la coexistencia de otros problemas médicos y el uso de múltiples medicaciones, hace a los ancianos más vulnerables para presentar sintomatología depresiva ${ }^{1}$.

Existen múltiples instrumentos de evaluación de trastornos mentales ${ }^{5}$ que pueden facilitar la identificación de casos o probables casos de depresión, resultando de utilidad clínica, aunque en ningún momento deben sustituir al juicio clínico, que será el que confirme el diagnóstico. La mayoría de las escalas y recomendaciones de entrevista para el estudio de la depresión ${ }^{5-8}$, no deberían se aplicadas en los ancianos porque no están adaptadas para ellos, lo que puede favorecer que se detecten un excesivo número de falsos positivos ${ }^{1,2}$.

Una de las escalas más utilizadas en la actualidad en población anciana es el cuestionario Geria tric Depression Scale de Yesavage (GDS), ${ }^{9}$, escala $^{10}$ también recomendada por la Sociedad Española de Medicina Familiar y Comunitaria ${ }^{11}$. Se trata de una escala autoaplicable de 30 preguntas con respuestas dicotómicas (sí/no) específicamente diseñada para la población anciana, con una elevada sensibilidad y especificidad $(\mathrm{S}=84 \% \text { y } \mathrm{E}=95 \%)^{9,10,12,13}$ y con una buena correlación con otras escalas clásicas utilizadas para el cribado de depresión ${ }^{14}$. Existe una validación al castellano ${ }^{15}$.

Sheikh y Yesavage ${ }^{16}$ propusieron posteriormente una versión abreviada de la GDS, formada por 15 preguntas (10 positivas y 5 negativas) que sólo requiere de 5 a 7 minutos para ser completada, con el objeto de reducir los problemas de fatiga y pérdida de atención que se suelen presentar en este grupo de edad, y más en los casos en que existe algún grado de alteración cognitiva. Aunque algunos autores han encontrado una correlación positiva, pero insuficiente, entre la versión corta y larga $(r=0,66)^{17}$, en otros trabajos la correlación encontrada fue superior al $0,80^{17,18} \mathrm{y}$ con datos de sensibilidad y especificidad equiparables entre las dos versiones ${ }^{18}$.

Este trabajo tiene como objetivo realizar la adaptación transcultural y validación de la GDS en su versión corta de 15 preguntas por considerarla un instrumento útil en el despistaje de depresión en población mayor de 65 años.

\section{SUJETOS, MATERIAL Y MÉTODOS}

\section{Sujetos}

El tamaño de la muestra necesario fue fijado en 250 personas (intervalo de confianza del $95 \%$ y $\alpha=0,05$ ), para así satisfacer los requerimientos de tamaño de muestra recomendados por algunos autores para este tipo de trabajos ${ }^{19,20}$, si bien se amplió un $10 \%$ en previsión de las posibles pérdidas. La población de estudio ha estado formada por hombres y mujeres mayores de 65 años que acudieron a las consultas del Centro de Salud de Azahara (Córdoba), tuvieran o no un diagnóstico previo de 
depresión. Los sujetos fueron reclutados de las consultas médicas y de enfermería de forma aleatoria hasta alcanzar el número de sujetos requerido. A todos los candidatos se les informó sobre el objetivo del estudio para que decidieran si deseaban participar en el mismo. Se incluyeron a personas mayores de 65 años, que aceptaron ser incluídos en el trabajo y que no presentaran ninguno de los criteriso de exclusión. Se consideraron criterios de exclusión: pacientes con alteración psiquiátrica grave no depresiva o con déficit motor y/o sensorial severo que impidiese la evaluación prevista. La recogida de datos se realizó a lo largo del año 1999.

\section{Material}

Además del cuestionario objeto de la validación, se utilizaron los siguientes instrumentos:

-Para la valoración de depresión: cuestionario semiestructurado para detectar síntomas depresivos, así como la escala de Montgomery-Asberg ${ }^{21}$.

-Para la valoración del estado cognitivo: estudio neuropsicológico, basado en el protocolo para diagnóstico de demencia de la Sociedad Española de Neurología ${ }^{22}$, así como los cuestionarios de Pfeiffer $^{23}$ en su versión española (SPMSQ-VE) ${ }^{24}$, el Mini-examen cognoscitivo de Lobo (MEC) $)^{25,26}$ y el test del informador en su versión española (IQCODE-VE) ${ }^{27}$.

-Para la valoración de las actividades de la vida diaria, básicas e instrumentales, se utilizaron las preguntas correspondientes de la escala OARSMFAQ (Older Americans Resources and Services Multidimensional Functional Assessment Ques tionnaire) en su versión española ${ }^{28}$.

Con toda esta información se establecieron los diagnósticos de depresión, de demencia, de ausencia de sintomatología psicopatológica o de duda diagnóstica, siguiendo los criterios de la Clasificación Internacional de Enfermedades (CIE-10) ${ }^{29}$.

Se consideró como "caso" a cualquir sujeto con diagnóstico clínico de depresión según criterios CIE-10 independientemente del tipo y del grado de severidad de la misma.

\section{Métodos}

\section{Adaptación transcultural}

Procedimos a la adaptación transcultural, siguiendo los siguientes pasos ${ }^{30-32}$ :

1. Puesta en contacto con el autor del cuestionario original, quien nos autorizó a llevar a cabo la adaptación y validación de la escala.

2. Traducción del texto original (del inglés al español) por tres personas bilingües con expe- riencia en la asistencia médica, que trabajaron por separado.

3. Retrotraducción (de la primera versión en castellano al inglés) por tres traductores nativos que desconocían tanto la existencia del original en inglés, como el hecho de que otras personas retrotraducían al mismo tiempo.

4. Formación de un comité revisor, integrado por los investigadores y los traductores, para comparar las retrotraducciones con el original inglés. Los criterios de comparación fueron: a) literales, si el significado global en las dos versiones y los cambios de palabras fueron los mismos; b) semejantes, si hubo cambios en el significado de alguna palabra, pero no en el conjunto de la pregunta; c) diferentes, si se encontró pérdida del significado original; y d) cambio en la pregunta, cuando se requirieron cambios en la formulación para adaptarlos a nuestra cultura.

5. Se dirimieron las discrepancias mediante la revisión de los enunciados según criterios de los investigadores y de los retrotraductores, tras la comparación del original con la retrotraducción y la revisión de los ítems. Finalmente, después de haber entrevistado a una muestra piloto de 20 personas, se concretó la versión definitiva (GDS-VE).

\section{Validación}

Una vez realizada la adaptación, en una primera fase, se administró el cuestionario a los participantes en el estudio. A pesar de que el cuestionario original es autoaplicado, en nuestro estudio -debido al elevado número de analfabetos- fue cumplimentado en todos los casos por profesionales médicos y de enfermería, a los que se les informó de los objetivos y metodología del trabajo y a los que únicamente se les facilitó las instrucciones propuestas por Yesavage, sin someterles a un entrenamiento previo.

Estudio de la fiabilidad: para analizar la fiabilidad interobservador, dos observadores distintos, de forma independiente y en el mismo momento, evaluaron a treinta sujetos con el GDS-VE. Para establecer la fiabilidad intraobservador, cincuenta sujetos fueron citados a los 7-10 días de la primera evaluación para volverles a aplicar el cuestionario por el mismo profesional.

Estudio de la validez: a ciegas y de forma independiente de la puntuación obtenida con el GDSVE, a todos los sujetos del estudio se les realizó un examen clínico y neuropsicológico protocolizado (especificado anteriormente) para establecer o no el diagnóstico de depresión, constituyendo este diagnóstico el patrón oro, con el que establecimos la validez del cuestionario que nos ocupa (Fig. 1). Esta evaluación fue realizada por dos psiquiatras y tres médicos de familia, previamente entrenados y supervisados éstos por los dos psiquiatras. Antes 


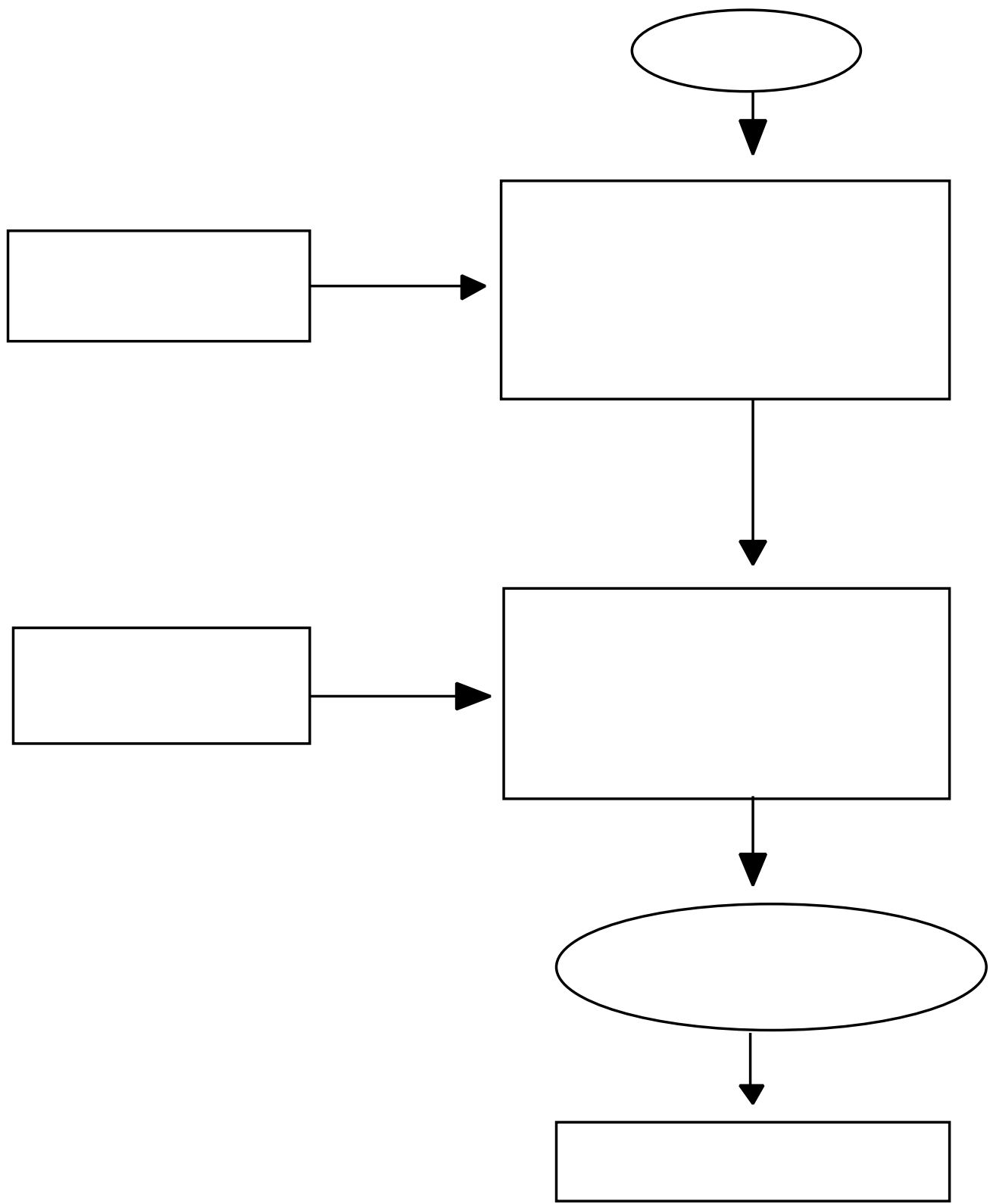

del inicio del estudio se realizaron sesiones conjuntas con los médicos evaluadores, en las que se analizaron distintos pacientes con el fin de homogeneizar los criterios diagnósticos. Posteriormente, se realizaron 20 grabaciones en video de entrevistas reales para medir la corcondancia interobservador de los 5 médicos evaluadores, alcanzando ésta un índice de kappa ponderado de 0,83 .

Una vez iniciada la recogida de datos, cuando existió alguna duda sobre la presencia o no de depresión, el caso fue discutido entre el grupo evaluador, estableciéndose posteriormente el diagnóstico final. 


\section{ANÁLISIS DE LOS DATOS}

Se ha utilizado el paquete estadístico SPSS-9.0. Se realizó un análisis descriptivo de las características sociodemográficas y de los diagnósticos utilizando medidas de tendencia central y distribución de frecuencias, calculando los intervalos de confianza (IC) para un 95\% de seguridad. Para establecer la asociación entre variables se calculó la jicuadrado para las cualitativas y la $t$ de Student y ANOVA para las cuantitativas.

Para evaluar la fiabilidad del cuestionario se analizaron 3 aspectos: fiabilidad interobservador e intraobservador mediante el cálculo del Kappa ponderado de Cohen (al no seguir una distribución normal la puntuación del cuestionario) y la consis tencia interna a través del estadístico de Kuder-Richarson-20 (KR-20).

Para evaluar la validez se estudió la validez de constructo y la de criterio. Validación de construc to: en primer lugar se examinó la validez convergente, valorando la correlación existente entre el GDS-VE y la escala de Montgomery-Asberg de detección de depresión. En segundo lugar, se examinó la validez discriminante, comparando los resultados del GDS-VE con la puntuación obtenida al aplicar a los mismos sujetos el cuestionario de SPMSQ-VE para despistaje de alteración cognitiva, en ambos casos se calculó el coeficiente de correlación de Spearman.

Validación de criterio o práctica (grado en que los resultados del test se correlacionan con el diagnóstico de depresión según los criterios CIE-10): para ello se calculó la sensibilidad (S), especificidad (E) y las razones de máxima verosimilitud para los resultados positivo y negativo, o razones de probabilidad positiva (RPP) y negativa (RPN) del cuestionario, así como el porcentaje de casos mal clasificados (falsos positivos o negativos). La capacidad discriminativa del cuestionario para diferenciar los sujetos sanos de los que tenían depresión se analizó mediante el área bajo la curva ROC (recei ver operating characteristic).

\section{RESULTADOS}

En el anexo 1 se muestra la adaptación transcultural realizada de la versión original. Con respecto a los criterios de comparación entre la versión original y la retrotraducción al inglés de la adaptación al castellano, en las preguntas $3,7,8,9,13,15$ éstos fueron literales, en la $1,2,4,5,6,10,11$ y 14 fueron semejantes; únicamente la pregunta 12 fue diferente.

Se captaron 280 sujetos de los cuales finalizaron el estudio 249. Las pérdidas fueron en 23 casos por rechazo a acudir a la segunda parte del estudio, 5 por presentar demencia severa y otros 3 por diversas causas (dificultades de comprensión, fallecimiento e ingreso en una residencia). La media de edad fue de 74,3 años (DE=6,5; rango: 65-100), siendo el 67,5\% mujeres. El 44,2\% estaban casados y el $37,8 \%$ viudos, residiendo el $72,7 \%$ en su propio domicilio. El $65 \%$ era analfabeto o tan solo sabía leer y escribir (Tabla I). Noventa casos $(36,1 \%)$ se diagnosticaron de depresión, tras la entrevista psiquiátrica. La puntuación media obtenida en la escala GDS-VE fue de 4,7 (DE:3,5, rango: 0-14), superior en la mujeres $(5,5 ; \mathrm{DE}: 3,6)$ que en los hombres $(2,9 ; \mathrm{DE}: 2,4)$, sin detectarse diferencias en relación a los grupos de edad ni nivel de estudios (Tabla I).

Resultados de fiabilidad: la fiabilidad interobservador e intraobservador, medidas a través del índice de Kappa ponderado, obtuvo valores de 0,655 $(p<0,001)$ y $0,951(p<0,001)$ respectivamente, para un punto de corte de 5 o más. La consistencia interna alcanzó un valor de 0,994 .

Resultados de validez: la validez convergente alcanzó un coeficiente de correlación de Spearman de 0,618 (p<0,001) al comparar el GDS-VE con los resultados del cuestionario de Montgomery-Asberg. En la validez discriminante, que se obtuvo al correlacionar la puntuación del GDS-VE con la obtenida con el SPMSQ-VE, el coeficiente de correlación de Spearman fue de 0,235 ( $p<0,001)$.

En la figura 2 se presenta la curva ROC del cuestionario validado, que obtuvo un área bajo la curva de 0,835 (IC:0,78-0,89), obteniéndose la mejor relación sensibilidad/especificidad para un punto de corte de 5. Posteriormente se agruparon los casos en función de que presentaran o no algún grado de alteración cognitiva. En el grupo de pacientes con alteración cognitiva $(\mathrm{n}=57)$, el área bajo la curva obtenida fue de 0,745 (IC:0,616-0,874). En el grupo de pacientes sin alteración cognitiva $(\mathrm{n}=192)$ el área obtenida fue de 0,865 (IC:0,809$0,921)$. En ambos casos el punto de corte con mayor área bajo la curva se sitúa en 5 .

En la tabla II se reflejan los datos de sensibilidad, especificidad, razón de probabilidad positiva y negativa para diferentes puntos de corte y el porcentaje de pacientes mal clasificados para el total de la muestra. En la tabla III se muestran los mismos resultados diferenciando los pacientes según tuvieran o no alteración cognitiva.

$\mathrm{Al}$ analizar los resultados de cada una de las preguntas de forma aislada, se ha podido observar que la preguntas 3 y 4 son las que obtienen mayor área bajo la curva (0,726 y 0,697 respectivamente), y las de menor área las preguntas 9 y 11 (Tabla IV).

\section{DISCUSIÓN}

La patología depresiva en el paciente anciano presenta una prevalencia e incidencia elevadas, re- 


\section{Anexol}

ESCALA DE DEPRESIÓN GERIÁTRICA DE YESAVAGE ABREVIADA (Versión española): GDS-VE

1. ¿ En general, está satisfecho/ a con su vida?

2. ¿Ha abandonado muchas de sus tareas habituales y aficiones?

3. ¿ Siente que su vida está vacía?

4. ¿Se siente con frecuencia aburrido/a?

5. ¿Se encuentra de buen humor la mayor parte del tiempo?

6. ¿Teme que algo malo pueda ocurrirle?

7. ¿Se siente feliz la mayor parte del tiempo?

8. ¿Con frecuencia se siente desamparado/a, desprotegido/ a?

9. ¿Prefiere usted quedarse en casa, más que salir y hacer cosas nuevas?

10. ¿Cree que tiene más problemas de memoria que la mayoría de la gente?

11. ¿En estos momentos, piensa que es estupendo estar vivo?

12. ¿Actualmente se siente un/ a inútil?

13. ¿ Se siente lleno/ a de energía?

14. ¿Se siente sin esperanza en este momento?

15. ¿Piensa que la mayoría de la gente está en mejor situación que usted?

PUNTUACIÓN TOTAL

Las respuestas que indican depresión están en negrita. Cada una de estas respuestas cuenta un punto.

\section{Tabla I}

CARACTERÍSTICAS SOCIODEM OGRÁFICAS DE LA MUESTRA Y PUNTUACIONES OBTENIDAS EN EL GDS-VE

\begin{tabular}{|c|c|c|c|c|c|}
\hline & $\begin{array}{l}\text { Población } \\
\text { estudio } \\
\text { n (\%) }\end{array}$ & $\begin{array}{c}\text { Puntuación media } \\
\text { y desviación estándar } \\
\text { en el GDS-VE }\end{array}$ & $p$ & $\begin{array}{l}\text { Diagnóstico } \\
\text { depresión } \\
\text { (CIE-10) }\end{array}$ & $p$ \\
\hline \multicolumn{6}{|l|}{ Sexo } \\
\hline Mujer & $168(67,5)$ & $5,5(3,6)$ & 0,0001 & $76(45,2)$ & 0,0001 \\
\hline Hombre & $81(32,5)$ & $2,9(2,4)$ & & $14(17,3)$ & \\
\hline \multicolumn{6}{|l|}{ Grupos de edad } \\
\hline $65-69$ & $65(26,1)$ & $5,1(3,7)$ & & $31(47,7)$ & \\
\hline $70-74$ & $78(31,3)$ & $4,9(3,7)$ & 0,21 & $28(35,9)$ & 0,02 \\
\hline $75-79$ & $52(20,9)$ & $4,6(3,4)$ & & $20(38,5)$ & \\
\hline$>79$ & $54(21,7)$ & $3,8(2,7)$ & & $11(20,4)$ & \\
\hline \multicolumn{6}{|l|}{ Nivel de estudios } \\
\hline Analfabeto & $85(34,1)$ & $4,8(3,5)$ & & $34(41,5)$ & \\
\hline Lee y escribe & $77(30,9)$ & $4,8(3,3)$ & 0,45 & $27(35,1)$ & 0,29 \\
\hline Estudios primarios o más & $87(34,9)$ & $4,2(3,4)$ & & $26(29,9)$ & \\
\hline
\end{tabular}

GDS-VE: Escala geriátrica de depresión. Versión española. 


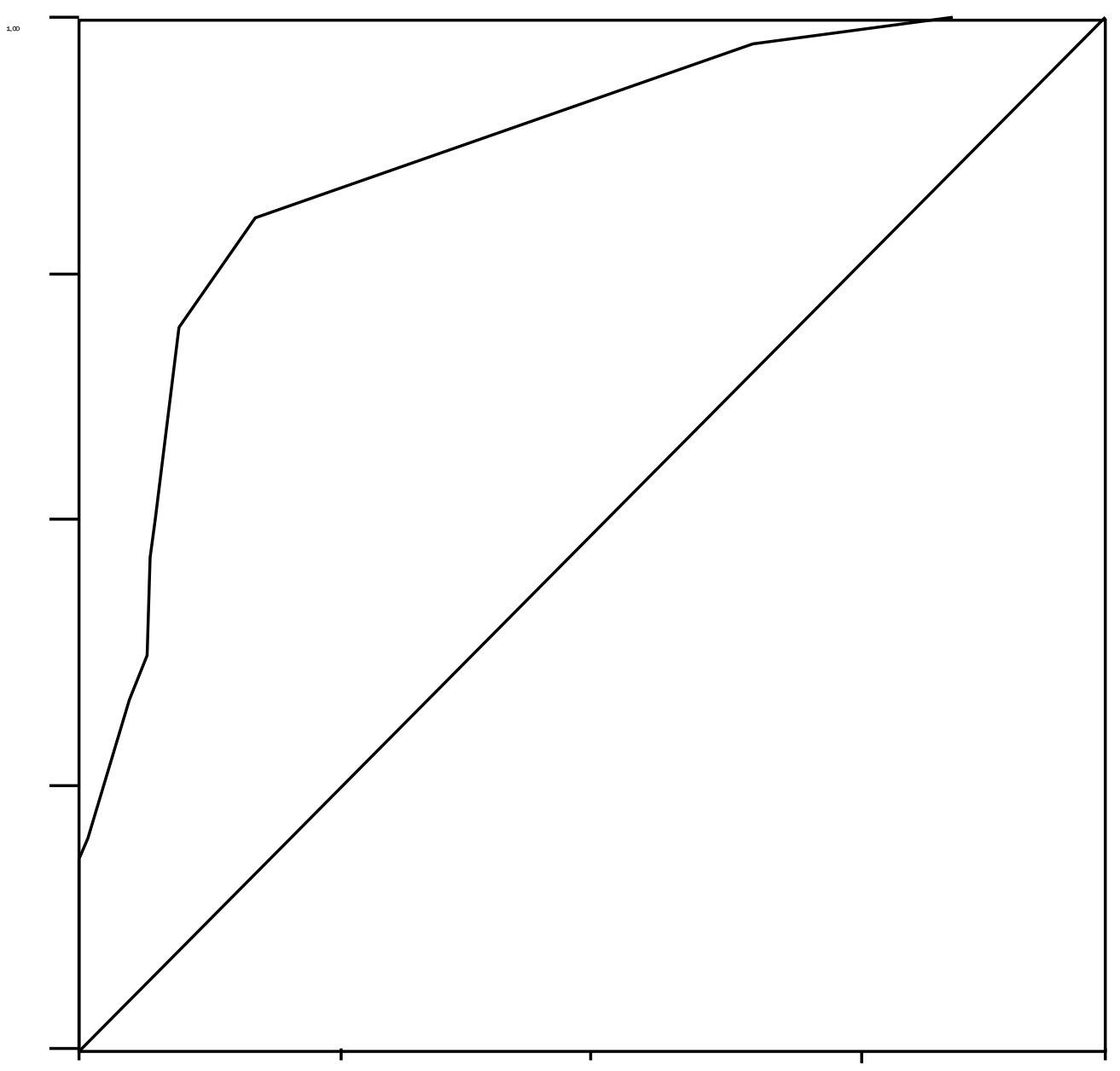

presentando una importante carga de malestar para el paciente, para la familia y para las instituciones en donde reside. Las características con las que se presenta la enfermedad en este grupo de edad, hacen que se diagnostique poco y se trate menos ${ }^{33}$. Estudios longitudinales han comprobado que la presencia de síntomas depresivos en ancianos se asocian con un mayor número de visitas al médico, de uso de fármacos, de utilización de servicios de urgencias y de costes globales como pacientes ambulatorios ${ }^{34,35}$.
El personal sanitario de Atención Primaria (tanto médicos/as como enfermeros/as) se encuentran situados en el nivel de atención, relación y accesibilidad idóneo para establecer con mayor facilidad el estado mental de las personas mayores, tanto desde el punto de vista afectivo como cognitivo ${ }^{36}$, pudiendo favorecer el diagnóstico precoz de los trastornos depresivos.

Para facilitar la detección de los cuadros depresivos, son de mucha utilidad cuestionarios que sean breves, fiables y de fácil utilización por parte del 
Tabla II

PARÁMETROS DE VALIDEZ SEGÚN PUNTO DE CORTE DEL GDS-VE, EN PACIENTES CON Y SIN ALTERACIÓN COGNITIVA ( $n=249)$

\begin{tabular}{lcccc} 
& $\geq 4$ & $\geq 5$ & $\geq 6$ & $\geq 7$ \\
\hline Sensibilidad & 86,7 & 81,1 & 72,6 & 51,1 \\
Especificidad & 61,6 & 76,7 & 85,5 & 90,6 \\
Razón de probabilidad positiva & 2,2 & 3,5 & 5,0 & 5,3 \\
Razón de probabilidad negativa & 0,2 & 0,2 & 0,3 & 0,5 \\
Porcentaje de pacientes mal clasificados & 29,3 & 21,7 & 20,9 & 23,7 \\
\hline
\end{tabular}

GDS-VE: Escala geriátrica de depresión. Versión española.

Tabla III

PARÁMETROS DE VALIDEZ SEGÚN PUNTO DE CORTE DEL GDS-VE EN FUNCIÓN DE LA PRESENCIA DE ALTERACIÓN COGNITIVA

PACIENTES SIN NINGÚN GRADO DE ALTERACIÓN COGNITIVA ( $n=192$ )

\begin{tabular}{lcccc}
\hline & $\geq 4$ & $\geq 5$ & $\geq 6$ & $\geq 7$ \\
\hline Sensibilidad & 91,2 & 86,8 & 72,1 & 52,9 \\
Especificidad & 61,3 & 77,4 & 87,1 & 91,9 \\
Razón de probabilidad positiva & 2,4 & 3,8 & 5,6 & 6,5 \\
Razón de probabilidad negativa & 0,1 & 0,2 & 0,3 & 0,5 \\
Porcentaje de pacientes mal clasificados & 28,1 & 19,3 & 18,2 & 21,9 \\
\hline
\end{tabular}

PACIENTES CON NINGÚN GRADO DE ALTERACIÓN COGNITIVA ( $n=57)$

\begin{tabular}{lcccc}
\hline & $\geq 4$ & $\geq 5$ & $\geq 6$ & $\geq 7$ \\
\hline Sensibilidad & 72,2 & 63,6 & 54,5 & 45,5 \\
Especificidad & 62,9 & 74,3 & 80,0 & 85,7 \\
Razón de probabilidad positiva & 1,9 & 2,5 & 2,7 & 3,2 \\
Razón de probabilidad negativa & 0,4 & 0,4 & 0,6 & 0,6 \\
Porcentaje de pacientes mal clasificados & 33,3 & 29,8 & 29,8 & 29,8 \\
\hline
\end{tabular}

GDS-VE: Escala geriátrica de depresión. Versión española.

personal de Atención Primaria. En el ámbito de la depresión en el paciente anciano, como ya se ha comentado anteriormente, es recomendable poder contar con cuestionarios enfocados a las particularidades de presentación de este cuadro en las personas mayores. Por este motivo hemos seleccionado el cuestionario de Yesavage en su versión breve, para su validación en nuestro medio.

En lo referente a la adaptación, no se han tenido que aplicar cambios importantes en el contenido del cuestionario y tan sólo la pregunta 12 sufrió una variación importante en su redacción.

Con respecto a los sujetos incluídos, el elevado porcentaje de personas analfabetas o sin ningún nivel de escolarización, que alcanzó el $65 \%$, no difiere mucho de los datos del nivel de escolarización de la población mayor de nuestro entorno ${ }^{37-39}$. Este dato fue lo que nos inclinó a no utilizar la escala de forma autoaplicada, como ya se ha comentado en el apartado de material y método, asumiendo las ventajas e inconvenientes que esto puede tener ${ }^{40}$.

Los resultados de fiabilidad de la GDS-VE han sido bastante satisfactorios, sobre todo teniendo en cuenta que se ha usado el índice de Kappa ponderado que es más exigente que el coeficiente de correlación intraclase, con el que se hubieran obteni- 
Tabla IV

SENSIBILIDAD, ESPECIFICIDAD Y AUC DE CADA PREGUNTA

\begin{tabular}{lccc} 
GDS-VE & S & E & AUC \\
\hline 1. ¿En general, está satisfecho/ a con su vida? & 31,1 & 91,2 & 0,612 \\
2. ¿Ha abandonado muchas de sus tareas habituales y aficiones? & 37,8 & 79,2 & 0,585 \\
3. ¿Siente que su vida está vacía? & 71,1 & 74,1 & 0,726 \\
4. ¿Se siente con frecuencia aburrido/ a? & 63,3 & 76,1 & 0,697 \\
5. ¿Se encuentra de buen humor la mayor parte del tiempo? & 56,7 & 79,9 & 0,683 \\
6. ¿Teme que algo malo pueda ocurrirle? & 60,0 & 66,0 & 0,630 \\
7. ¿Se siente feliz la mayor parte del tiempo? & 44,4 & 86,8 & 0,656 \\
8. ¿Con frecuencia se siente desamparado/ a, desprotegido/ a? & 48,9 & 87,4 & 0,682 \\
9. ¿Prefiere usted quedarse en casa, más que salir y hacer cosas nuevas? & 63,3 & 46,8 & 0,551 \\
10. ¿Cree que tiene más problemas de memoria que la mayoría de la gente? & 35,6 & 79,9 & 0,577 \\
11. ¿En estos momentos, piensa que es estupendo estar vivo? & 20,0 & 91,2 & 0,556 \\
12. ¿Actualmente se siente un/ a inútil? & 38,9 & 82,4 & 0,606 \\
13. ¿ Se siente lleno/ a de energía? & 54,4 & 78,0 & 0,662 \\
14. ¿Se siente sin esperanza en este momento? & 50,0 & 74,8 & 0,624 \\
15. ¿Piensa que la mayoría de la gente está en mejor situación que usted? & 43,3 & 85,5 & 0,644
\end{tabular}

S: Sensibilidad. E: Especificidad. AUC: Área bajo la curva ROC. GDS-VE: Escala geriátrica de depresión. Versión española.

do valores de fiabilidad intra e interobservador de 0,96 y 0,89 respectivamente, considerados como excelentes. El hecho de que las puntuaciones obtenidas en el GDS-VE no presentaran una distribución normal, nos ha inclinado a utilizar el primer método a pesar de sus peores resultados ${ }^{41}$. Por otro lado, la consistencia interna obtenida fue muy elevada. Todo esto sugiere que la capacidad de reproductibilidad del cuestionario es alta ${ }^{19}$.

La obtención de un área bajo la curva de 0,835 , representa la buena capacidad que tiene la escala para diferenciar las personas mayores con depresión de las que no la presentan ${ }^{42}$. El punto de corte con mejor relación sensibilidad/especificidad ha sido el de 5 o más puntos, tal y como recomiendan los autores del cuestionario original ${ }^{11}$, obteniendo una sensibilidad y especificidad de 81,1 y 76,7 respectivamente, resultados similares a los de otros trabajos que utilizaron la versión original ${ }^{18,43}$. A pesar de todo, con este punto de corte el GDS-VE, clasifica mal a 2 de cada 10 pacientes, resultados algo mejores a los obtenidos por $\mathrm{Cwikel}^{44}$. Hay que recordar que tanto éste como otros cuestionarios no deben ser considerados nunca como pruebas diagnósticas, si no como métodos de cribado rápido y, en todo caso, como una ayuda a la clasificación de pacientes susceptibles de ser evaluados con criterios más fiables.

Una de las ventajas del GDS, tanto en su versión de 30 preguntas como en esta de 15 , consiste en sus buenos resultados, incluso en pacientes con alteración cognitiva. En este trabajo se han evaluado los resultados de validez de forma global, pero también diferenciando en dos grupos a la población analizada, en función de que presentaran o no algún grado de alteración cognitiva. Ésta fue establecida mediante la cumplimentación de un estudio neuropsicológico ${ }^{22}$. Los pacientes con alteración cognitiva muy importante, que impedía la comprensión de la escala, fueron excluídos del trabajo. Como se puede observar en la tabla III, los resultados son obviamente mejores en aquellos casos en los que no se detectó ninguna alteración cognitiva. En los pacientes en los que se detectó algún grado de alteración cognitiva, el cuestionario permitió etiquetar adecuadamente a más del $70 \%$ de los casos, alcanzando la especificidad valores muy similares a los de la versión inglesa ${ }^{18}$.

Al analizar la validez de cada pregunta de forma individualizada, se ha comprobado que las preguntas número $3,4,5$ y 8 son las que presentan una mayor capacidad de discriminar entre posibles depresivos y sanos. Varios autores han propuesto escalas aún más cortas para el cribado de la depresión, basadas en el GDS abreviado ${ }^{45-47}$ que cuentan con 10, 5, 4 o incluso con tan sólo una pregunta. Holy et al, han validado una versión de 5 preguntas extraídas de la GDS abreviada, con aparentemente 
buenos resultados de validez. De estas cinco preguntas seleccionadas dos de ellas coinciden con alguna de las cuatro que en este estudio han presentado mejores resultados. La pregunta número 3 (¿siente que su vida está vacía?), que es la que presenta mayor área bajo la curva, ha sido propuesta como pregunta única para detectar de forma simple síntomas de depresión ${ }^{46}$. De cualquier forma parece que son precisos estudios más amplios para poder recomendar el uso generalizado de estas escalas "ultracortas".

Para finalizar, se debe hacer mención a que la aplicación del cuestionario se realizó por personal médico y de enfermería que no había recibido ningún tipo de entrenamiento previo (únicamente las instrucciones adjuntas al cuestionario) y en las consultas normales de demanda/programada. Este hecho, que puede haber empeorado los resultados finales (dada la posible variabilidad inducida por la falta de entrenamiento previo), lo consideramos como positivo, ya que lo que intentamos fue obtener los resultados de validez en situaciones lo más "reales" posibles. Por otro lado, una de las limitaciones del trabajo se sitúa en el hecho de que no se ha evaluado la sensibilidad al cambio del cuestionario, lo cual limita su uso en estudios longitudinales ${ }^{48}$. Por otro lado, la forma de obtención de la muestra, puede sugerir un sesgo de selección, aunque dadas las características del estudio (validación de un cuestionario) no parece que haya influído excesivamente en los resultados, ya que se trata de pobla- ción general de consulta que no difería en sus características sociodemográficas de la población andaluza ni española, tal y como se ha comentado anteriormente.

Con demasiada frecuencia se utilizan cuestionarios inapropiados y sobre todo no validados en nuestro medio. El hecho de poder contar con un cuestionario de la brevedad del GDS-VE, para su utilización en la práctica habitual en Atención Primaria, y que además por su sencillez pueda ser usado por todo tipo de personal sanitario, puede hacer que el despistaje y posterior diagnóstico de depresión en los pacientes ancianos sea más efectivo.

Se puede consultar ésta y otras versiones en:

http://wwwleland.stanford.edu/ yesavage/GDS.ht $\mathrm{ml}$

\section{AGRADECIMIENTOS}

A todos los profesionales del Centro de Salud de Azahara y a los pacientes que aceptaron participar en este estudio.

\section{CORRESPONDENCIA:}

Jorge Martínez de la Iglesia

C/ Samuel Santos Gener, 3

14003 Córdoba

\section{Bibliografía}

1. Calcedo Barba A. La depresión en el Anciano. Doce cuestiones fundamentales. Madrid: Fundación Archivos de Neurobiología, 1996.

2. García L, Nebreda O, Perlado F. Enfermedad mental en el anciano. Madrid; Ed. Díaz de Santos, 1993.

3. Feighner JP, Boyer WF. Diagnóstico de la depresión. Perspectivas en Psiquiatría (vol.2). Madrid: Ed Wiley, 1991.

4. Calcedo Barba A. La detección de trastornos psiquiátricos en Atención Primaria. En: López Ibor JJ. Libro del Año Psiquiatría. Madrid: Saned, 1995.

5. Bulbena A, Fernández de Larrinoa P, Pomarol E. Instrumentos de evaluación de los trastornos mentales en atención primaria. En: Vázquez-Barquero, editor. Psiquiatría en atención primaria. Madrid: Aula Médica, 1998. p. 131-49.

6. Wetzler S, Van Praag HM. Evaluación de la depresión. En: Medición de las enfermedades mentales: evaluación psicométrica para los clínicos. New York: Ancora, 1991.

7. McDowell Y, Newell CL. Depression. En: Measuring Health. A guide to rating scales and questionnaires. London: Oxford University Press, 1996.

8. BrinK TL, Yesavage JA, Lum O, Heersema PH, Adey M, Rose T. Screening tests for geriatric depression. Clinical
Gerontologist 1982; 1 (1): 37-43.

9. Yesavage JA, BrinK TL, Rose TL, Lum O. Development and validation of a geriatric depression scale: a preliminary report. J Psychiat Res 1983; 17 (1): 37-49.

10. Yesavage JA. Geriatric Depression scales. Psychopharmacol Bull 1988; 24: 709.

11. Grupo de trabajo de la Sociedad Española de Medicina Familiar y Comunitaria. Atención al anciano. Madrid: Ed. Eurobook SL., 1997.

12. Van MarwijK H, Arnold Y, Bonnema J, Kaptein A. Selfreport depression scales for elderly patients in primary care: a preliminary study. Fam Pract 1993; 10 (1): 63-5.

13. McGivney SA, Mulvihil M, Taylor B. Validating the GDS depression screen in the nursing home. J Am Geriatr Soc 1994; 42: 490-2.

14. Salamero M, Marcos T. Factor study of the Geriatric Depression Scale. Acta Psychiatr Scand 1992; 86: 2836

15. Izal M, Montorio I. Adaptación en nuestro medio de la Escala de Depresión Geriátrica (GDS) en distintos subgrupos: residentes en la comunidad y asistentes a hospitales de día. Rev Gerontol 1996; 6: 329-37. 
16. SheiKh JL, Yesavage JA. Geriatric Depression Scale (GDS). Recent evidence and development of a shorter version. Clin Gerontol 1986; 5: 165-72.

17. Alden D, Austin C, Sturgeon R. A correlation between the Geriatric Depression Scale Long and Short Forms. J Gerontol Psychol Sc 1989; 44 (4): 124-5.

18. Lesher EL, Berryhill JS. Validation of a Geriatric Depression Scale-short form among inpatients. J Clinic Psychol 1994; 50 (2): 256-60.

19. Grau G. Metodología para la validación de cuestionarios. MEDIFAM 1995; 5: 351-9.

20. Kline P. A handbook of test construction. London; Methuen, 1986.

21. Montgomery SA, Asberg H. A new depresion scale designed to be more sensitive to change. Br J Psychiatr 1979; 134: 382-9.

22. Grupo de estudio de Neurología de la conducta y demencias. SEN. Guía en demencias. Barcelona: Masson, 2000.

23. Pfeiffer E. A Short Portable Mental Status Questionaire for the assesment of organic brain deficit in elderly patients. $\mathbf{J}$ Am Geriatr Soc 1975; 23: 433-41.

24. Martínez J, Dueñas R, Onís MC, Aguado C, Albert C, Luque R. Adaptación y validación al castellano del cuestionario de Pfeiffer (SPMSQ) para detectar la existencia de deterioro cognitivo en personas mayores de 65 años. Med Clin (Barc) 2001; 117: 129-34.

25. Lobo A, Ezquerra J, Gómez F, Sala JM, Seva A. El miniexamen cognoscitivo (un "test" sencillo, práctico, para detectar alteraciones intelectuales en pacientes médicos). Actas Luso Esp Neurol Psiquiatr 1977; 7: 189-202.

26. Lobo A, Saz P, Marcos G, Día JL, de la Cámara C, Ventura $\mathrm{T}$, et al. Revalidación y normalización del Mini-Examen Cognoscitivo (primera versión en castellano del Mini-Mental Status Examination) en población general geriátrica. Med Clin (Barc) 1999; 112: 767-74.

27. Morales JM, González JI, del Ser T, Bermejo F. El test del informador. Una nueva aproximación en la detección precoz de la demencia. Rev Esp Geriatr Gerontol 1993; 28: 142-53.

28. Grau,G, Eiroa P, Cayuela A. Versión española del OARS multidimensional Functional Assessment Questionnaire: Adaptación transcultural y medida de la validez. Aten Primaria 1996; 17:486-95.

29. CIE 10. Trastornos mentales y del comportamiento. Descripciones clínicas y pautas de comportamiento. Organización Mundial de la Salud. Madrid: Meditor, 1992.

30. Guillemin F, Bombardier C, Beaton D. Cross-cultural adaptation of health-related quality of life measures: literature review and proposed guidelines. J Clin Epidemiol 1993; 46: 1417-32.

31. Agra I. Adaptación transcultural de cuestionarios en ciencias de la salud. Medifam 1997; 7: 13-6.

32. Guillemin F. Cross cultural adaptation and validation of health status measures. Scand J Rheumatol 1995; 24: 61-3.

33. Lebowitz BD, Pearson JL, Schneider LS, Reynols III CF, Alexopoulos GS, Bruce ML, et al. Actualización y trata- miento de la depresión en los ancianos. Actualización de los informes del consenso. JAMA (ed esp) 1998; 7: 162-7.

34. Cooper-Patrick L, Crum RM, Ford DE. Characteristics of patients with major depression who received care in general medical and speciality health settings. Med Care 1994; 32 : 15-24.

35. Callahna CM, Hui SL, Nienaber NA, Musick BS, Tierney WM. Longitudinal study of depression and health services use among elderly primary care patients. J Am Geriatr Soc 1994; 42: 833-8.

36. Fernández Viadero C. Trastornos mentales habituales en ancianos. En: Vázquez-Barquero JL, ed. Psiquiatría en atención primaria. Madrid: Aula Médica, 1998. p. 477-94.

37. Dirección General de atención Sanitaria. Guía de atención a la salud del anciano. Sevilla; Junta de Andalucía. Consejería de Salud, 1992.

38. Galat VL, Zunzunegui MV, Béland F. El diseño y la ejecución de "Envejecer en Leganés". Rev Gerontol 1995; 5: 215-31.

39. Martínez de la Iglesia J, Pérula de Torres LA, Espejo J, Rubio MV, Aranda JM, Fonseca FJ, et al. Proyecto ANCO: un estudio sociosanitario de la población mayor de la ciudad de Córdoba. Diseño y ejecución. Rev Gerontol 1997; 7: 8290.

40. De la Serna de Pedro, I. Manual de psicogeriatría clínica. Barcelona: Masson, 2000.

41. Prieto L, Lamarca R, Casado A. La evaluación de la fiabilidad en las observaciones clínicas: el coeficiente de correlación intraclase. Med Clin (Barc) 1998; 110: 142-5.

42. Tobías A, Fernández F. Pruebas diagnósticas (III): curvas ROC. Jano 1999: LVI: 1090.

43. Almeida OP, Almeida SA. Short versions of the geriatric depression scale: a study of their validity for the diagnosis of a major depressive episode according to ICD-10 and DSM-IV. Int J Geriatr Psychiatry 1999; 14: 858-65.

44. Cwikel J, Ritchie K. Screening for depression among the elderly in Israel an assessment of the Short Geriatric Depression Scale (S-GDS). Isr J Med Sci 1989; 25 (3): 1317.

45. Holy MT, Alessi CA, Harker JO, Josephson KR, Pietruszka FM, Koelfgen M, et al. Development and testing of a fiveitem version of the Geriatric Depression Scale. Am Geriatr Soc 1999; 47: 873-8.

46. D'Ath p, Katona P, Mullan E, Evans S, Katona C. Screening, detection and management of depression in elderly primary care attenders. I: the acceptability and performance of the 15 item Geriatric Depression Scale (GDS15) and the development of short versions. Fam Pract 1994; 11: 260-6.

47. Clement JP, Nassif RF, Leger JM, Marchan F. Development and contribution to the validation of brief French version of the Yesavage Geriatric Depression Scale. Encephale 1997; 23: 91-9.

48. Badía X, Baró E. Cuestionarios de salud en España y su uso en atención primaria. Aten Primaria 2001; 28: 349-56. 